\title{
Glycerol and neutral lipid production in the oleaginous marine diatom Phaeodactylum tricornutum promoted by overexpression of glycerol-3-phosphate dehydrogenase
}

\author{
Yao Yao ${ }^{\dagger}$, Yang Lu ${ }^{\dagger}$, Kun-Tao Peng, Tan Huang, Ying-Fang Niu, Wei-Hong Xie, Wei-Dong Yang, Jie-Sheng Liu \\ and Hong-Ye Li
}

\begin{abstract}
Background: Microalgae are ideal raw materials for biodiesel and bioactive compounds. Glycerol-3-phosphate is formed from dihydroxyacetone phosphate (DHAP) through the glycolytic pathway catalyzed by glycerol-3-phosphate dehydrogenase (GPDH).

Results: GPDH was characterized in the marine diatom Phaeodactylum tricornutum. In the GPDH-overexpressing P. tricornutum cells, the glycerol concentration per cell in the transformed diatom increased by 6.8 -fold compared with the wild type, indicating that the overexpression of GPDH promoted the conversion of DHAP to glycerol-3-phosphate. There was a $60 \%$ increase in neutral lipid content, reaching $39.7 \%$ of dry cell weight in transgenic cells in the stationary phase, despite a $20 \%$ decrease in cell concentration. Fatty acid profiling showed that the levels of 16- and 18-carbon monounsaturated fatty acids significantly increased.

Conclusion: GPDH had a significant impact on numerous metabolic processes in diatom cells, including the biosynthesis of glycerol and neutral lipids. These findings are instructive for the metabolic engineering of microalgae for biofuel production.
\end{abstract}

Keywords: Glycerol-3-phosphate dehydrogenase, Glycerol, Diatom, Lipid

\section{Background}

It has become increasingly clear that conventional fossil fuels, such as coal, petroleum, and natural gas, are not sustainable in respect to operational efficiency and environmental impact due to the requirement for enormous consumption of these fuels and the greenhouse gas emissions caused by them [1]. To solve the problem of energy supply and environmental concerns caused by the consumption of fossil fuels, alternative renewable biofuels must be developed [2,3]. Vegetable oils and animal fats were the first generation of biofuels [4], but these still cannot meet the growing demand from the rapidly developing world. The energy and acreage required for oil-producing

\footnotetext{
* Correspondence: thyli@jnu.edu.cn

${ }^{\dagger}$ Equal contributors

Key Laboratory of Eutrophication and Red Tide Prevention of Guangdong

Higher Education Institutes, College of Life Science, Jinan University,
} Guangzhou 510632, China plants have been the limiting factors for biofuel development [5]. Thus, the attention of scientists has been shifted from the land to the ocean, where microalgae are widely distributed and are initially responsible for ocean primary productivity.

In many marine microalgae species, lipids are the major storage metabolite and are generally seen in the form of triacylglycerols (TAGs) stored in oil bodies. Microalgae use light more efficiently and have a higher growth rate than terrestrial plants; moreover, they do not compete with agricultural crops for arable land. Therefore, microalgae are considered a promising candidate for renewable biofuel production $[6,7]$.

TAGs consist of a glycerol backbone and fatty acids (FAs); thus, glycerol is an important raw material for TAG synthesis. Glycerol-3-phosphate dehydrogenase (GPDH) is part of an important pathway in which the reduction of 
dihydroxyacetone phosphate (DHAP) from the glycolytic pathway is catalyzed into sn-glycerol-3-phosphate (sn, stereospecific numbering) in a reversible manner [8]. In the yeast Saccharomyces cerevisiae, the isoenzymes of NAD-dependent GPDH are encoded by two homologous genes, GPD1 and GPD2 $[9,10]$. GPD1 was found to play a role in osmoadaptation, as its expression was induced by osmotic stress and in osmosensitive gpd1 delta mutants. In cells exposed to increased external osmolarity, expression of GPD1 is elevated and leads to increased production and accumulation of intracellular glycerol [10-12]. A yeast GPD1 gene was expressed in transgenic oil-seed rape seed driven by a seed-specific napin promoter, resulting in a $40 \%$ increase in lipid content, accompanied by a doubling of GPDH activity and a three fold to four-fold increase in glycerol-3-phosphate [13].

GPDH is also referred to as DHAP reductase in algae and higher plants. Klöck et al. purified a GPDH enzyme from the green microalga Chlamydomonas reinhardtii, and found that it reacted specifically with NADH and DHAP as substrates [14]. Gee et al. separated three isoforms of DHAP reductase from Dunaliella tertiolecta, and concluded that two isoforms from Dunaliella chloroplasts played roles in glycerol production and glyceride synthesis, respectively [15]. In Dunaliella salina, NAD ${ }^{+}$ -dependent GPDH can directly catalyze the conversion from DHAP to glycerol, and it has an extra 300-amino acid fragment that harbors the phosphoserine phosphatase domain [16]. Relative to the study of GPD isozyme genes in S. cerevisiae and higher plants, microalgal GPD studies have focused only on the separation and purification of the natural protein. However, in microalgae, the characteristics of GPD isozyme genes, or of their expression and function, have rarely been reported.

Diatoms are major primary producers in the marine ecosystem. Phaeodactylum tricornutum, a diatom species, is rich in proteins and lipids, and has a fast growth rate and an available genome sequence (http://genome.jgi-psf. org/Phatr2/Phatr2.home.html). Thus, these attributes have made $P$. tricornutum a promising candidate for genetic modification of its traits for the mass commercial production of bioproducts. Owing to the important role of GPDH in lipid metabolism and glycerol production, its role in the molecular mechanisms of diatoms must be characterized. So far, no investigation into the GPDH of diatoms has been reported, and its function remains unclear. Thus, in this study, GPDH of P. tricornutum was characterized in transgenic $P$. tricornutum to determine its function.

\section{Results}

Sequence prediction of GPDH

The putative GPDH gene of P. tricornutum (GenBank accession: XP_002177310.1) was obtained by BLAST searching on
NCBI, based on the GPDH gene of S. cerevisiae (GenBank accession number Z24454). The amino acid sequence of the putative GPDH protein of P. tricornutum showed high similarity with the GPDH protein from a range of species retrieved from GenBank. The phylogenetic tree of GPDH drawn with the software MEGA5 using the neighborjoining method [17] presented the similar type of phylogenetic tree as that using maximum likelihood and minimum-evolution methods (see Additional file 1: Figure S1) which adopted the same bootstrap method and Poisson correction model. As shown in Figure 1, the phylogenetic tree demonstrated that the GPDH cloned in this study shared higher homology with its counterparts from the marine diatom Thalassiosira spp. and the marine brown alga Ectocarpus siliculosus, which represented closer relationships.

\section{Molecular analysis of transgenic microalgae by genomic $\mathrm{PCR}$, quantitative $\mathrm{PCR}$, and western blotting}

Genomic PCR analysis was conducted to verify the transgene incorporation. A band of the expected size of $1.0 \mathrm{~kb}$ amplified with primers specific to the both ends of the $G P D H$ coding region using the transgenic line (Figure 2A, lane 3). Another pair of primers flanking both ends of $G P D H$ on the transformation vector was used to check the incorporation of the transgene. A slightly larger band was present from the transgenic cells (Figure 2A, lane 2), whereas no similar band was present from the wildtype cells (Figure 2A, lane 1). This result suggested that the $G P D H$ transgene was already incorporated into the transgenic diatom cells. The mRNA expression of the GPDH gene was demonstrated by quantitative PCR (qPCR) (Figure $2 \mathrm{~B})$. Using $\beta$-actin as a housekeeping marker, the relative expression level of GPDH in transgenic diatom cells was elevated threefold compared with that of the wild type. This result demonstrated that the introduced $G P D H$ was transcriptionally expressed, and consequently increased the GPDH transcript abundance in the transgenic diatom cells. The GPDH cloned in the transformation vector was fused with a C-terminal Myc tag (amino acids: EQKLISEEDL) to allow subsequent determination of heterologous protein expression. Thus, the heterologous GPDH protein in the transgenic microalgae was detected as a $37 \mathrm{kDa}$ band that cross-reacted with antiMyc antibodies in western blotting analyses (Figure 2C, lane 1), whereas no such band was present in the wild type (Figure 2C, lane 2).

\section{Measurement of the glycerol content}

A standard curve was first drawn using standard glycerol samples (Figure 3A). This standard curve, representing optical density (OD) on the $y$ axis against concentration of glycerol on the $x$ axis, was based on the absorbance of standard glycerol samples of different concentrations at 


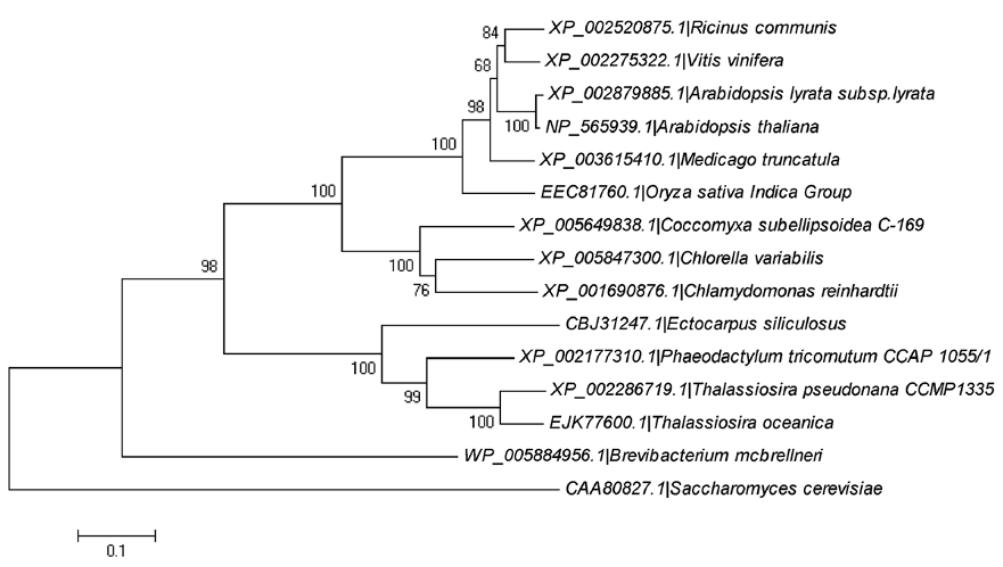

Figure 1 Phylogenetic tree based on the multiple alignment of glycerol-3-phosphate dehydrogenase (GPDH) protein sequences from Phaeodactylum tricornutum with related species. The tree was constructed using the neighbor-joining method. The bootstrap percentage shown at the nodes, as the estimator of accuracy, is based on a 1,000-replicate bootstrap analysis.

wavelength of $550 \mathrm{~nm}$. The curve could be converted to the equation $y=0.0075 x+0.0497$, and the R-squared value was 0.9993 , indicating the curve had a high degree of accuracy. The absorbance of the samples was put into the equation to obtain the concentration of the samples.

The glycerol content of transgenic diatom cells was $2.06 \times 10^{-10} \mu \mathrm{mol} / \mathrm{cell}$ and that of the wild type was $3.05 \times$ $10^{-11} \mu \mathrm{mol} / \mathrm{cell}$ (Figure 3B). Thus, the glycerol content in GPDH-overexpressing cells was increased by 6.8 -fold compared with the wild type. This result demonstrated that GPDH overexpression led to the increased conversion of dihydroxyacetone phosphate to glycerol-3-phosphate.

To detect whether overproduced glycerol was secreted from the diatom cells, the glycerol content in the culture medium was also measured, and showed similar glycerol concentrations in the extracellular medium of the transgenic diatom cells and the wild type (Figure 3B). Thus, the overproduced glycerol in transgenic diatom cells was not secreted out of the cells but rather was stored inside the cell or partially participated in downstream metabolic pathways.

\section{Analysis of the growth, lipid content, and fatty acid profile}

The growth rate of the transgenic diatom cells was slightly slower than that of the wild-type control (Figure 4A). The neutral lipid content was measured by Nile red staining, which showed that the neutral lipid content in the transgenic $P$. tricornutum cells had increased significantly up to 1.9-fold compared with wild-type cells (Figure 4B). These findings indicate that the transgenic diatom cells accumulated neutral lipid with a resulting detrimental effect on cell biomass. The lipid content was also assessed by gravimetric analysis, which showed that the lipid content in the transformed $P$. tricornutum significantly increased by $1.6-$
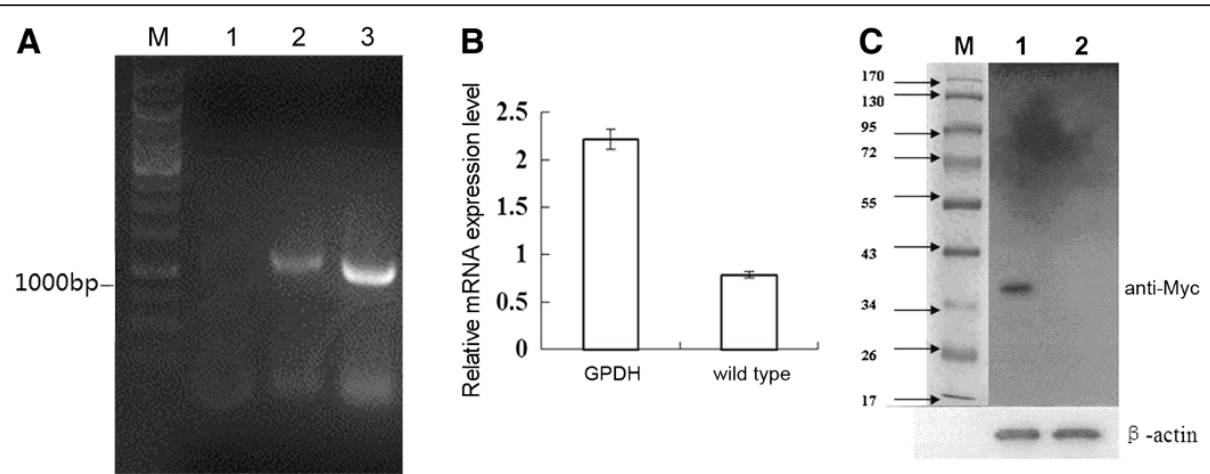

Figure 2 Molecular analysis of GPDH transgenic diatom cells. (A) Transgenic diatom cells selected in medium containing chloramphenicol were first tested by genomic PCR. Lane M: 1 kb plus DNA ladder; lane 1: PCR of wild type with primers on the transformation vector; lane 2: PCR of transgenic line with primers on the transformation vector flanking GPDH; lane 3: PCR of transgenic line with primers on both ends of GPDH. (B) GPDH mRNA expression in the diatom cells determined by quantitative PCR. (C) Protein expression of introduced GPDH detected by western blotting with anti-Myc antibody (Invitrogen, USA). M: Protein molecular weight marker; lane 1: transgenic P. tricornutum overexpressing GPDH tagged with Myc; lane 2: wild type. 

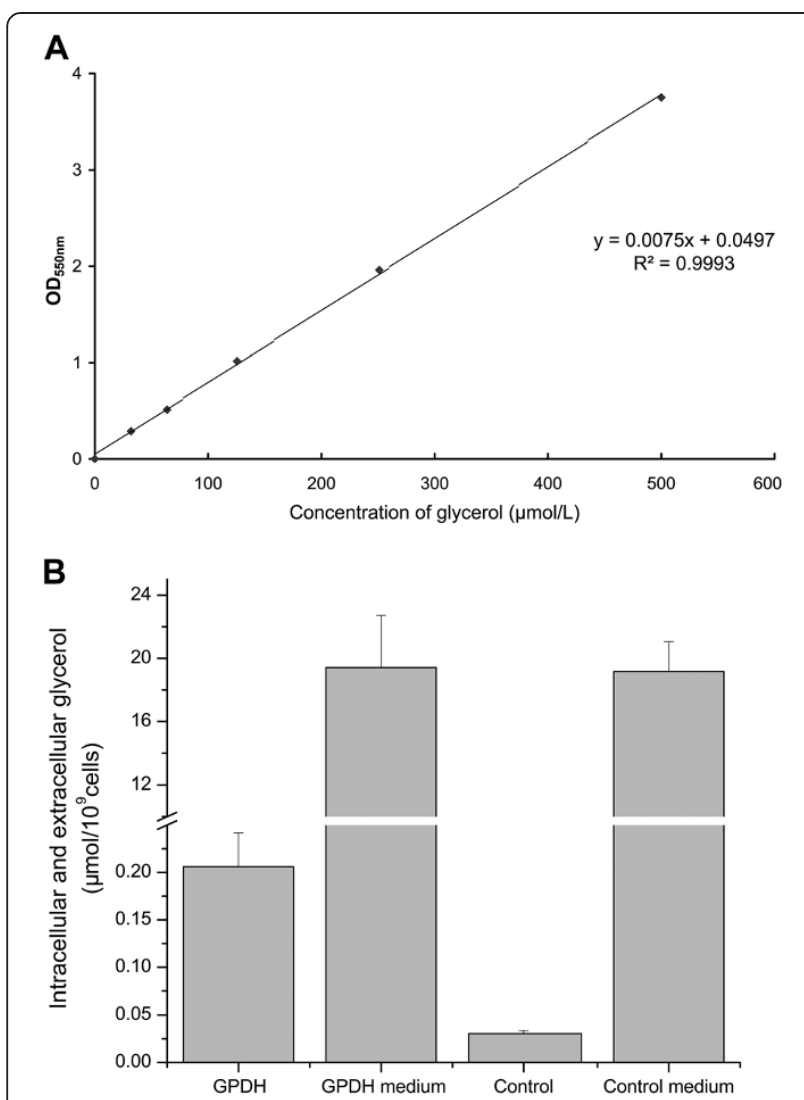

Figure 3 Glycerol content measurement in diatom cells. (A)

Standard curve for the glycerol concentration and its opacity density. (B) Intracellular glycerol content and glycerol concentration in the culture medium.

fold compared with wild-type cells (Figure 4C). The dry weight ratio between the lipid content in the transgenic cells and lyophilized algae powder was up to $39.7 \%$, whereas in wild-type cells, this ratio was only $24.8 \%$.

As shown in Table 1, there were significant changes in FA profile in GPDH-overexpressing transgenic diatom cells. The overall level of monounsaturated fatty acids (MUFAs) significantly increased. In particular, the content of C16:1 was almost doubled, and the content of C18:1 was threefold higher than that in the wild type. However, the polyunsaturated fatty acids (PUFAs) in transgenic cells, such as C16:3, C18:2 and C20:5, decreased by $56 \%, 30 \%$, and $24 \%$, respectively, compared with the wild type. The overall PUFA content decreased by $33 \%$. As for the saturated fatty acids (SFAs) in transgenic cells, only $\mathrm{C} 16: 0$ showed a significant change, with an increase of $21 \%$, whereas the other FAs were not altered significantly.

Diatom cells were further examined under a confocal laser-scanning microscope to detect morphological changes. Transgenic cells exhibited larger and had denser oil bodies compared with the wild type, and also had slightly higher fluorescence of oil bodies with Nile red staining (Figure 5).

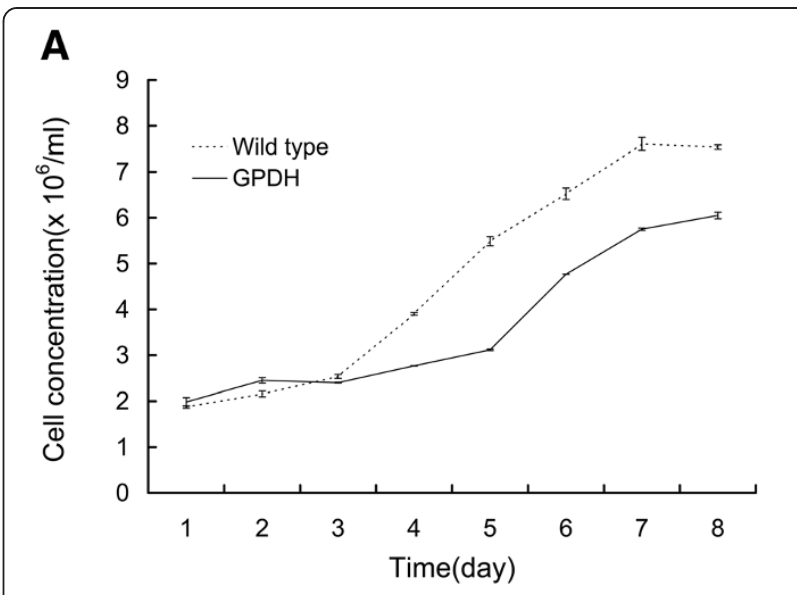

\section{B}

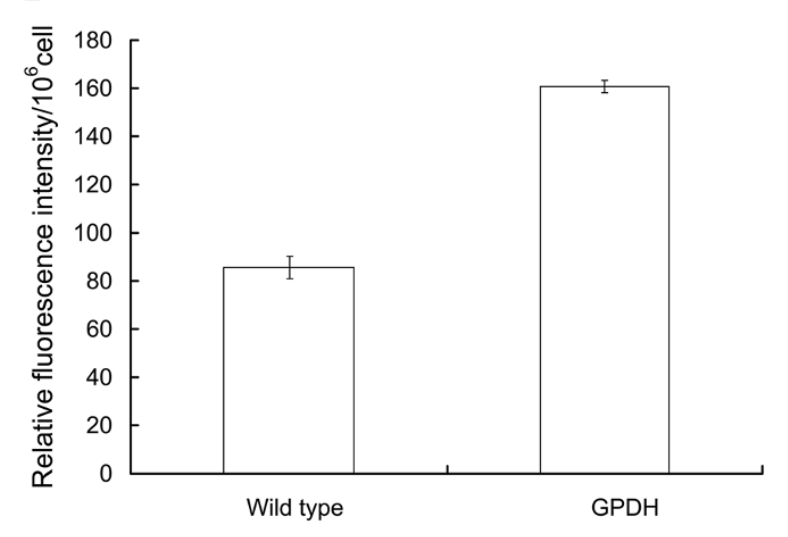

C

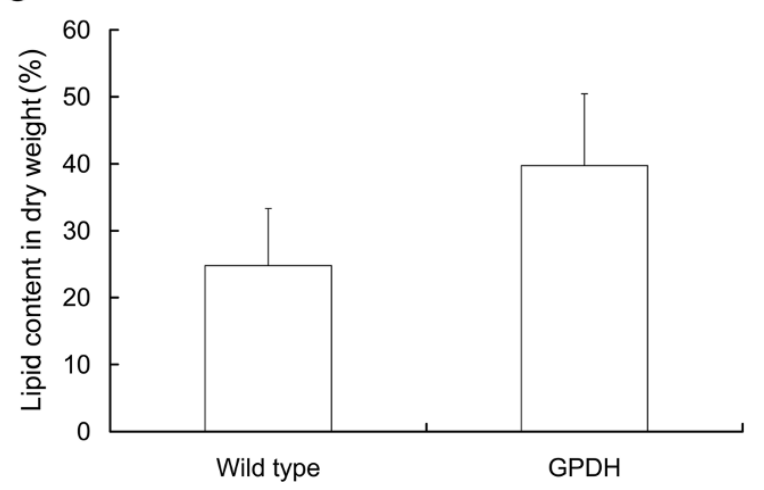

Figure 4 Growth and neutral lipid content of diatom cells.

(A) Cell concentration measured in a culture cycle; (B) neutral lipid content detected by Nile red staining in the stationary phase; (C) lipid content measured gravimetrically in the stationary phase.

\section{Discussion}

With the rapid progress of genome sequencing and transcriptome information, algal biofuel research has been gaining momentum. The configuration of core metabolic pathways in microalgae is highly variable between distinct algal classes. A major challenge in developing 'ideal' microalgal strains for biofuel production is the optimization of 
Table 1 Fatty acid composition in diatom cells

\begin{tabular}{lcc}
\hline Fatty acid & GPDH & Wild type \\
\hline $12: 0$ & 0.22 & 0.21 \\
$14: 0$ & 5.69 & 5.40 \\
$15: 0$ & 0.56 & - \\
$16: 0$ & 21.49 & 17.75 \\
17:0 & 0.10 & 0.12 \\
18:0 & 6.36 & 7.04 \\
20:0 & 0.10 & 0.17 \\
22:0 & 0.03 & - \\
24:0 & 0.07 & 0.33 \\
Total SFA & 34.62 & 31.02 \\
16:1 & 33.11 & 17.81 \\
18:1 & 4.43 & 1.47 \\
Total MUFA & 37.54 & 19.28 \\
16:3 & 2.41 & 5.46 \\
18:2 & 1.46 & 2.09 \\
20:5 & 8.97 & 11.78 \\
20:2 & 0.08 & 0.07 \\
22:6 & 0.57 & 0.51 \\
Total PUFA & 13.49 & 19.91 \\
\hline MUFA monounsara & & $5 F A$
\end{tabular}

MUFA, monounsaturated fatty acid; PUFA, polyunsaturated fatty acid; SFA, saturated fatty acid. Values are in percentage of total fatty acids.

the accumulation of both biomass and fuel-relevant molecules, such as TAG. Selecting targets for genetic manipulation requires a fundamental understanding of microalgae and regulation of their carbon metabolic pathways. A goal of many studies is to identify gene targets for genetic manipulation to improve microalgal growth and characteristics leading to lipid, or more specifically, TAG accumulation, thus driving down the cost of biofuel production $[18,19]$.

In this work, we characterized a glycerol-3-phosphate dehydrogenase, which was demonstrated to play a role in regulating glycerol production and lipid accumulation in $P$. tricornutum. The nitrate reductase promoter and fcp (fucoxanthin chlorophyll a/c-binding proteins) promoter used in the expression vector achieved successful transcription of heterologous genes, thereby allowing the selection of transformed diatom cells and the expression of functional GPDH.

A previous study of the GPDH of $S$. cerevisiae specifically expressed in rapeseed (Brassica napus L.) seeds using seedspecific promoters showed that the content of FAs and lipids in seeds increased with no change in the FA composition [13]. Although rapeseed, which is a higher plant, is markedly different from the single-celled phytoplankton $P$. tricornutum, the current work showed that overexpression of GPDH in P. tricornutum also increased the content of neutral lipids. The overproduced glycerol was not fully used in TAG biosynthesis, as that the content of glycerol increased 6.8-fold, whereas the content of neutral lipids increased 1.9-fold. In higher plants such as oil-seed rape, precursors can be acquired from other tissues to serve as the source materials for neutral lipid biosynthesis and as substances to regulate the activity of ACCase (acetyl-CoA carboxylase) $[13,20]$. In this study, P. tricornutum, as a single-celled phytoplankton, was not able to mobilize precursor materials from other cells; however, the synthesis of glycerol-3-phosphate that was promoted in the GPDH-overexpressing diatom cells led to an increase in the lipid content, as in the case of rapeseed. Thus, it suggests that the overexpression of GPDH in $P$. tricornutum enhanced the synthesis of glycerol and neutral lipids, and that GPDH plays an important role in lipid accumulation in diatoms.

Based on the measurement of glycerol, most of the produced intracellular glycerol was not secreted from the cells. In the case of the overproduced glycerol in the cell, it was usually secreted to maintain the osmolality equilibrium, which is regulated by Fps1p, a type of channel protein on the cell membrane [21]. An explanation of our findings is that glycerol mainly exists in the form of glycerol-3phosphate, and the increased neutral lipid content indicates that the GPDH of P. tricornutum plays a similar role to that of $S$. cerevisiae, andpromoted the biosynthesis of neutral lipids. In S. cerevisiae, there are two isoenzymes of GPDH, called GPD1 and GPD2, whose cofactors are NAD. GPD1 is found in the cytoplasm, and GPD2 in the plastid. The former facilitates FA accumulation, whereas the latter is responsible for the synthesis of membrane lipids and signal transduction lipids [9].

In high salt-stress environments, halophilic algae appear to be efficient converters which can convert stored starch rapidly into a large amount of glycerol within the cells to adapt to external changes under osmotic pressure [22]. GPDH, a key enzyme in the synthetic route of glycerol, acts as a precursor for both glycerol and lipids in halophilic microalgae. Putative GPDH genes from Chlamydomonas reinhardtii, including $C r G P D H 1$, $\mathrm{CrGPDH} 2$, and $\mathrm{CrGPDH}$, show a high sequence similarity with GPDH from D. salina and Dunaliella viridis, and exhibited increased expression of $\mathrm{CrGPDH} 2$ and CrGPDH3 in response to osmotic stress, which was correlated with the accumulation of glycerol and TAG in this microalga [23,24]. Correspondingly, elevated expression of the GPDH genes of $D$. salina and $D$. viridis was detected under osmotic stress by $\mathrm{NaCl}[16,25]$. Our results were consistent with that in the above microalgae, thus presenting a base for further functional characterization of GPDH in microalgae at the biochemical and molecular levels.

Moreover, in our study, the profile of FAs in transgenic diatom cells exhibited some obvious changes in addition 


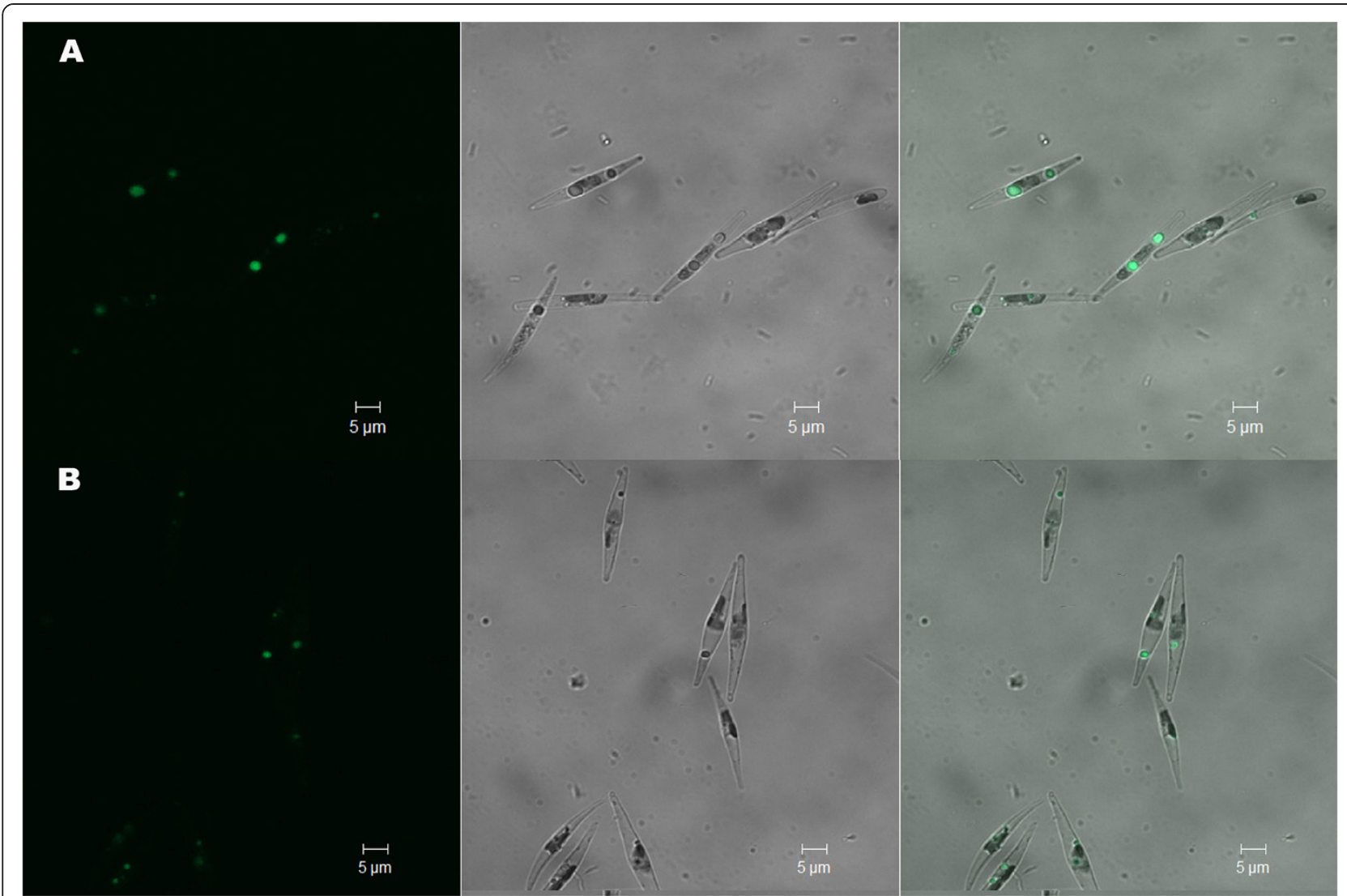

Figure 5 Nile red-stained diatom cells. Diatom cells in the stationary phase were stained with Nile red and photographed under a confocal microscope. (A) Transgenic cells; (B) Wild-type cells. Left: red fluorescence of oil bodies; middle: differential interference contrast (DIC); right: overlay image. Bars $=5 \mu \mathrm{m}$.

to the increase in the neutral lipid content. However, the reason for the increase in C16:1 and C18:1 MUFAs needs further study for clarification.

\section{Conclusion}

The identified novel diatom GPDH plays a role in various metabolic pathways. GPDH-overexpressing P. tricornutum cells showed an increase in the synthesis of glycerol and neutral lipids. The detailed biological functions of the diatom GPDH need to be further studied. The findings are also instructive for the genetic manipulation of microalgae for biofuel production.

\section{Materials and methods}

\section{Diatom strain and culture conditions}

P. tricornutum Bohlin was purchased from the Freshwater Algal culture Collection of the Institute of Hydrobiology, CAS, China (catalogue number FACHB-863), and was used for GPDH cloning and expression. The diatom cells were grown in batch cultures in $\mathrm{f} / 2$-Si medium in Erlenmeyer flasks, and were filter sterilized by $0.22 \mu \mathrm{m}$ filter membranes (Millipore, Billerica, MA, USA). P. tricornutum was cultivated periodically (8 days of a life cycle), and the preceding cultures were used as inocula. Cultures in liquid or solid media were grown at $21 \pm 1^{\circ} \mathrm{C}$ in an artificial climate incubator (Jiangnan, Ningbo, China) that was set with a $12 \mathrm{~h} / 12 \mathrm{~h}$ light/dark photoperiod. Cool-white fluorescent tubes provided a constant irradiance of $200 \mu \mathrm{mol}$ photons per $\mathrm{m}^{2}$ per second.

\section{GPDH cloning, vector construction, and diatom transformation}

GPDH of P. tricornutum was predicted by BLAST searching against GPDH of $S$. cerevisiae on the NCBI website. $P$. tricornutum cells were harvested by centrifuging at $3,000 \times \mathrm{g}$ for 10 minutes, and the total RNA was extracted with an RNAiso kit (TaKaRa, Dalian, China). GPDH cDNA was obtained by RT-PCR with the PrimeScript II 1st Strand cDNA Synthesis Kit (TaKaRa). The primers for GPDH amplification were Pt507 and Pt508 (Table 2).

$G P D H$ was cloned into a P. tricornutum transformation vector derived from the plasmid pHY11 [26]. Expression of GPDH was driven by an fcpC promoter/fcpA terminator cloned from $P$. tricornutum. Chloramphenicol was used as the selection marker under the $P$. tricornutum nitrate reductase promoter. $P$. tricornutum was transformed 
Table 2 List of primers used in this study

\begin{tabular}{ll}
\hline Primer name & Sequence \\
\hline Pt507 & ACCATGAAAGCATCTTCTCCACCTC \\
Pt508 & GTTCGTTCGTCAAAGTTCTCCATT \\
GPDHq1 & GCGACGATTGGCTTGACCT \\
GPDHq2 & TCCGTGGATGATGCTTTGATTCT \\
ACTf & AGGCAAAGCGTGGTGTTCTTA \\
ACTr & TCTGGGGAGCCTCAGTCAATA \\
\hline
\end{tabular}

with the GPDH expression vector by electroporation using a GenePulser Xcell apparatus (Bio-Rad, Hercules, CA, USA) following the protocol of Niu et al. [26]. After electroporation, $P$. tricornutum cells were transferred into liquid medium $(\mathrm{f} / 2-\mathrm{Si})$ and incubated for 24 hours in the dark. The transgenic cells were selected onf/2-Si solid selection medium supplemented with chloramphenicol. The surviving colonies were picked and transferred to liquid medium (f/2Si) with $250 \mathrm{mg} / \mathrm{l}$ chloramphenicol for further selection and successive culture every 8 days of a life cycle.

\section{Molecular analysis of the transgenic microalgae by genomic PCR, quantitative PCR, and western blotting} After continuous subculturing for five more cycles in solid selection medium, the transgenic cells were analyzed by a range of molecular approaches.

Genomic PCR was first used to screen the transformed cells to indicate the presence of heterologous GPDH gene. The primers used were Pt89a and Pt91r, which flanked the two ends of the GPDH gene on the vector. The genomic DNA of the diatom-transformed cells was extracted with the Universal Genomic DNA Extraction Kit (v.3.0; TaKaRa). PCR-confirmed cultures were then subjected to successive culture for further analysis.

To detect the mRNA expression level, qPCR was performed using SYBR Premix Ex Taq II kit (Takara) with primers GPDHq1 and GPDHq2. The reactions were conducted on a Bio-Rad CFX96 Real-Time PCR System. The putative $\beta$-actin (ACT1, Phatrdraft_51157) from $P$. tricornutum was used as a housekeeping marker, with forward primer ACTf and reverse primer ACTr. The threshold cycle for each well was determined, and the relative mRNA expression levels of GPDH were quantified after normalization to $\beta$-actin.

The expression of GPDH protein in transgenic diatom cells was determined by western blotting analysis. Approximately $200 \mathrm{ml}$ microalgae cells $\left(7 \times 10^{6}\right.$ cells $\left./ \mathrm{ml}\right)$, which were cultivated until the stationary phase, were collected by centrifugation at $3,000 \times \mathrm{g}$ for 10 minutes at $4{ }^{\circ} \mathrm{C}$. The total protein was extracted using a Protein Extraction Kit (KeyGEN, Nanjing, China), and the protein concentration was determined by a Protein Assay Kit (Bio-Rad). The total protein (40 $\mu \mathrm{g}$ per well) was separated by SDS-PAGE on a $10 \%$ gel and electrotransferred onto a
PVDF membrane (Millipore, Billerica, MA, USA). The membrane was blocked in PBS containing 5\% non-fat milk for 2 hour at $4{ }^{\circ} \mathrm{C}$ and then incubated with anti-Myc antibody (Invitrogen, Grand Island, NY, USA) at 1:5,000 dilution at $4^{\circ} \mathrm{C}$ overnight. The membrane was washed for 10 minutes with PBS-T (137 mM NaCl, $2.7 \mathrm{mM} \mathrm{KCl}$,

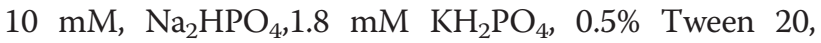
$\mathrm{pH}$ 7.6) three times, and incubated with HRP-conjugated goat anti-rabbit secondary antibody at 1:5,000 dilution (ComWin Biotech Ltd., Beijing, China) for 1 hour at room temperature. After washing three times with PBS-T, the membrane was visualized with the BeyoECL Plus Kit (Beyotime, Jiangsu, China) following the manufacturer's instructions.

\section{Analysis of the glycerol and neutral lipid contents in transgenic diatoms}

The lipid content in P. tricornutum was measured by Nile red (Sigma-Aldrich, St. Louis, MO, USA) staining [27]. Approximately $300 \mu \mathrm{l}$ diatom culture at the stationary phase and $3 \mu \mathrm{l}$ Nile red solution $(0.1 \mathrm{mg} / \mathrm{ml}$ in acetone solution) were mixed and stained for 20 minutes at room temperature. The fluorescence intensity of the stained cultures was measured by a microplate reader with a $530 \mathrm{~nm}$ excitation wavelength and a $580 \mathrm{~nm}$ emission wavelength. Unstained diatom culture and stained medium acted as the controls. The final fluorescence value was calculated by subtracting the fluorescence intensity value of the unstained diatom culture and the fluorescence value of the stained medium. The cell concentration of the algal culture was determined using a hemocytometer to obtain the cell concentration.

The glycerol content was quantitatively measured using a Glycerol Content GPO-POD Enzymatic Assay Kit (Applygen Technologies Inc., Beijing, China) following the manufacturer's manual. The OD detected by spectrophotometry had a positive linear association with the glycerol concentration. Therefore, the standard curve was drawn by spectrophotometry at $550 \mathrm{~nm}$ wavelength through the corresponding relationship between the OD value and the glycerol concentration.

\section{Observation of the morphological changes in the transgenic diatom}

The morphology of the transgenic microalgal cells was observed and photographed under a laser-scanning confocal microscope LSM 510 META (Zeiss, Oberkochen, Germany). Cell cultures were stained with Nile red $(0.1 \mathrm{mg} /$ $\mathrm{ml}$ in acetone) directly in a volume ratio of 1:100 (Nile red: cell culture) for approximately 20 minutes in the dark. The wavelengths for the fluorescence detection were $488 \mathrm{~nm}$ for excitation and 505 to $550 \mathrm{~nm}$ for emission. 


\section{Analysis of the fatty acid composition in the transgenic diatom}

Fatty acid composition was analyzed according to the protocol of Yang et al. [27]. In particular, diatom cells at the stationary phase were harvested by centrifugation at $3,000 \times \mathrm{g}$ for 10 minutes at $4^{\circ} \mathrm{C}$. FAs were extracted from the diatom cells, and the FA composition was analyzed by gas chromatography-mass spectrometry (GC-MS) (Finnigan TRACE DSQ; ThermoFisher, Waltham, MA, USA) at the Guangdong Institute of Microbiology. The integrated peak areas were determined and calculated after normalization to obtain the relative percentage of the FA profile.

\section{Gravimetric determination of the lipid content in $P$. tricornutum cells}

Approximately $250 \mathrm{ml}$ of diatom cells were cultivated for 8 days to the stationary phase, then collected by centrifugation at $3,000 \times \mathrm{g}$ for 10 minutes at $4{ }^{\circ} \mathrm{C}$ and lyophilized for 48 hours (MODULYOD-230, ThermoFisher). Approximately $20 \mathrm{mg}$ lyophilized algae powder was mixed with $2 \mathrm{ml}$ of methanol, $2 \mathrm{ml}$ of chloroform, and $1 \mathrm{ml}$ of $5 \% \mathrm{NaCl}$ [28]. According to the method of Huang et al. [29], the mixture was vortexed for $2 \mathrm{~min}$ and centrifuged at $8,000 \mathrm{~g}$ for $4 \mathrm{~min}$. The chloroform layer was collected, the procedure was repeated three times, and the combined extracts were dried by $\mathrm{N}_{2}$ airflow. The lipid remainder was then dried in an oven at $60^{\circ} \mathrm{C}$ and weighed on an electronic balance (with increments of $0.1 \mathrm{mg}$ ) (FA1004, Changzhou, China) to yield the dry weight.

\section{Additional file}

Additional file 1: Figure S1. Phylogenetic trees based on different methods of GPDH protein sequences from $P$. tricornutum with related species. (A) Maximum Likelihood method; (B) Minimum-Evolution method.

\section{Abbreviations \\ CrGPDH1: CrGPDH2, and CrGPDH3, Putative GPDH genes from Chlamydomonas reinhardtii; DHAP: Dihydroxyacetone phosphate; DIC: Differential interference contrast; GPDH: Glycerol-3-phosphate dehydrogenase; GC-MS: Gas chromatography-mass spectrometry; GPD1 , GPD2: Homologous genes encoding GPDH of Saccharomyces cerevisiae; MUFA: Monounsaturated fatty acids; PUFA: Polyunsaturated fatty acid; PVDF: polyvinylidene fluoride; GPCR: Quantitative real-time PCR; SFA: Saturated fatty acid; TAG: Triacylglycerols.}

\section{Competing interests}

The authors declare that they have no competing interests.

\section{Authors' contributions}

$Y Y, Y L Y N$ and $K P$ designed transformation vectors and generated transgenic diatoms. $Y Y, Y L, W X$ and KP maintained diatom cultures and performed molecular analysis. YY, YL, KP TH and WY analyzed the data. $\mathrm{HL}$ and $J \mathrm{~L}$ designed the experiments and wrote the article. All authors read and approved the final manuscript.

\section{Acknowledgements}

This work was supported by the National Science and Technology Program (2011BAD14B03), Department of Education of Guangdong (2013KJCX0024), Science and Technology Project of Guangdong (2010B030600005).

Received: 12 February 2014 Accepted: 8 July 2014

Published: 21 July 2014

\section{References}

1. Brennan L, Owende P: Biofuels from microalgae-A review of technologies for production, processing, and extractions of biofuels and co-products. Renew Sustain Energ Rev 2010, 14:557-577.

2. Hill J, Nelson E, Tilman D, Polasky S, Tiffany D: Environmental, economic, and energetic costs and benefits of biodiesel and ethanol biofuels. Proc Natl Acad Sci 2006, 103:11206-11210.

3. Adams IP, Dack S, Dickinson FM, Ratledge C: The distinctiveness of ATP: citrate lyase from Aspergillus nidulans. Biochim Biophys Acta 2002, 1597:36-41.

4. Aggelis G, Komaitis M, Papanikolaou S, Papadopoulos G: A mathematical model for the study of lipid accumulation in oleaginous microorganisms. I: Lipid accumulation during growth of Mucor circinelloides CBS 172-27 on a vegetable oil. Grasas y Aceites 1995, 46:169-173.

5. Antolın G, Tinaut F, Briceno Y, Castano V, Perez C, Ramırez A: Optimisation of biodiesel production by sunflower oil transesterification. Bioresour Technol 2002, 83:111-114.

6. Hu Q, Sommerfeld M, Jarvis E, Ghirardi M, Posewitz M, Seibert M, Darzins A: Microalgal triacylglycerols as feedstocks for biofuel production: perspectives and advances. Plant J 2008, 54:621-639.

7. Aliverti A, Pandini V, Pennati A, Rosa M, Zanetti G: Structural and functional diversity of ferredoxin-NADP ${ }^{+}$reductases. Arch Biochem Biophys 2008, 474:283-291.

8. Herrera-Valencia VA, Macario-Gonzalez LA, Casais-Molina ML, Beltran-Aguilar $A G$, Peraza-Echeverria S: In silico cloning and characterization of the glycerol-3-phosphate dehydrogenase (GPDH) gene family in the green microalga Chlamydomonas reinhardtii. Current microbiology 2012, 64:477-485

9. Ansell R, Granath K, Hohmann S, Thevelein JM, Adler L: The two isoenzymes for yeast $\mathrm{NAD}^{+}$-dependent glycerol 3-phosphate dehydrogenase encoded by GPD1 and GPD2 have distinct roles in osmoadaptation and redox regulation. EMBO J 1997, 16:2179-2187.

10. Eriksson $\mathrm{P}$, André $\mathrm{L}$, Ansell $\mathrm{R}$, Blomberg A, Adler L: Cloning and characterization of GPD2, a second gene encoding sn-glycerol 3-phosphate dehydrogenase $\left(\mathrm{NAD}^{+}\right)$in Saccharomyces cerevisiae, and its comparison with GPD1. Mol Microbiol 1995, 17:95-107.

11. Albertyn J, Hohmann S, Thevelein JM, Prior BA: GPD1, which encodes glycerol-3-phosphate dehydrogenase, is essential for growth under osmotic stress in Saccharomyces cerevisiae, and its expression is regulated by the high-osmolarity glycerol response pathway. Meth Cell Biol 1994, 14:4135-4144

12. Andre $L$, Hemming $A$, Adler $L$ : Osmoregulation in Saccharomyces cerevisiae studies on the osmotic induction of glycerol production and glycerol 3-phosphate dehydrogenase (NAD ${ }^{+}$). FEBS Letters 1991, 286:13-17.

13. Vigeolas H, Waldeck P, Zank T, Geigenberger P: Increasing seed oil content in oil-seed rape (Brassica napus L.) by over-expression of a yeast glycerol-3-phosphate dehydrogenase under the control of a seed-specific promoter. Plant Biotechnol J 2007, 5:431-441.

14. Klöck G, Kreuzberg K: Kinetic properties of a glycerol-3-phosphate dehydrogenase purified from the unicellular alga Chlamydomonas reinhardtii. Biochim Biophys Acta 1989, 991:347-352.

15. Gee R, Goyal A, Byerrum RU, Tolbert NE: Two isoforms of dihydroxyacetone phosphate reductase from the chloroplasts of Dunaliella tertiolecta. Plant Physiology 1993, 103:243-249.

16. He Q, Qiao D, Bai L, Zhang Q, Yang W, Li Q, Cao Y: Cloning and characterization of a plastidic glycerol 3-phosphate dehydrogenase cDNA from Dunaliella salina. J Plant Physiol 2007, 164:214-220.

17. Tamura K, Dudley J, Nei M, Kumar S: MEGA4: Molecular evolutionary genetics analysis (MEGA) software version 4.0. Mol Biol Evol 2007, 24:1596-1599.

18. Hildebrand M, Abbriano RM, Polle JE, Traller JC, Trentacoste EM, Smith SR, Davis AK: Metabolic and cellular organization in evolutionarily diverse 
microalgae as related to biofuels production. Curr Opin Chem Biol 2013, 17:506-514.

19. Smith SR, Abbriano RM, Hildebrand M: Comparative analysis of diatom genomes reveals substantial differences in the organization of carbon partitioning pathways. Algal Research 2012, 1:2-16.

20. Thelen JJ, Ohlrogge JB: Both antisense and sense expression of biotin carboxyl carrier protein isoform 2 inactivates the plastid acetyl-coenzyme A carboxylase in Arabidopsis thaliana. Plant J 2002, 32:419-431.

21. Remize F, Barnavon L, Dequin S: Glycerol export and glycerol-3-phosphate dehydrogenase, but not glycerol phosphatase, are rate limiting for glycerol production in Saccharomyces cerevisiae. Metab Eng 2001, 3:301-312.

22. Ben-Amotz A, Avron M: The role of glycerol in the osmotic regulation of the halophilic alga Dunaliella parva. Plant Physiology 1973, 51:875-878.

23. León R, Galván F: Halotolerance studies on Chlamydomonas reinhardtii: glycerol excretion by free and immobilized cells. J Appl Phycol 1994, 6:13-20.

24. Siaut M, Cuiné S, Cagnon C, Fessler B, Nguyen M, Carrier P, Beyly A, Beisson F, Triantaphylidès $C$, Li-Beisson Y: Oil accumulation in the model green alga Chlamydomonas reinhardtii: characterization, variability between common laboratory strains and relationship with starch reserves. BMC Biotechnol 2011, 11:7.

25. He Y, Meng X, Fan Q, Sun X, Xu Z, Song R: Cloning and characterization of two novel chloroplastic glycerol-3-phosphate dehydrogenases from Dunaliella viridis. Plant Mol Biol 2009, 71:193-205.

26. Niu YF, Zhang MH, Li DW, Yang WD, Liu JS, Bai WB, Li HY: Improvement of neutral lipid and polyunsaturated fatty acid biosynthesis by overexpressing a type 2 diacylglycerol acyltransferase in marine diatom Phaeodactylum tricornutum. Mar Drugs 2013, 11:4558-4569.

27. Yang Z, Niu Y, Ma Y, Xue J, Zhang M, Yang W, Liu J, Lu S, Guan Y, Li H: Molecular and cellular mechanisms of neutral lipid accumulation in diatom following nitrogen deprivation. Biotechnol Biofuels 2013, 6:67.

28. Ben-Amotz A, Tornabene TG, Thomas WH: Chemical profile of selected species of microalgae with emphasis on lipids. J Phycol 1985, 21:72-81.

29. Huang G-H, Chen G, Chen F: Rapid screening method for lipid production in alga based on Nile red fluorescence. Biomass Bioenergy 2009, 33:1386-1392.

doi:10.1186/1754-6834-7-110

Cite this article as: Yao et al: Glycerol and neutral lipid production in the oleaginous marine diatom Phaeodactylum tricornutum promoted by overexpression of glycerol-3-phosphate dehydrogenase. Biotechnology for Biofuels 2014 7:110.

\section{Submit your next manuscript to BioMed Central and take full advantage of:}

- Convenient online submission

- Thorough peer review

- No space constraints or color figure charges

- Immediate publication on acceptance

- Inclusion in PubMed, CAS, Scopus and Google Scholar

- Research which is freely available for redistribution 